\title{
Compact beam transport system for free-electron lasers driven by a laser plasma accelerator
}

\author{
Tao Liu, ${ }^{1,2,3}$ Tong Zhang, ${ }^{1}$ Dong Wang, ${ }^{1}$ and Zhirong Huang ${ }^{3}$ \\ ${ }^{1}$ Shanghai Institute of Applied Physics, Chinese Academy of Sciences, Shanghai 201800, China \\ ${ }^{2}$ University of Chinese Academy of Sciences, Beijing 100049, China \\ ${ }^{3}$ SLAC National Accelerator Laboratory, Menlo Park, California 94025, USA
}

(Received 1 September 2016; published 1 February 2017)

\begin{abstract}
Utilizing laser-driven plasma accelerators (LPAs) as a high-quality electron beam source is a promising approach to significantly downsize the x-ray free-electron laser (XFEL) facility. A multi-GeV LPA beam can be generated in several-centimeter acceleration distance, with a high peak current and a low transverse emittance, which will considerably benefit a compact FEL design. However, the large initial angular divergence and energy spread make it challenging to transport the beam and realize FEL radiation. In this paper, a novel design of beam transport system is proposed to maintain the superior features of the LPA beam and a transverse gradient undulator (TGU) is also adopted as an effective energy spread compensator to generate high-brilliance FEL radiation. Theoretical analysis and numerical simulations are presented based on a demonstration experiment with an electron energy of $380 \mathrm{MeV}$ and a radiation wavelength of $30 \mathrm{~nm}$.
\end{abstract}

DOI: 10.1103/PhysRevAccelBeams.20.020701

\section{INTRODUCTION}

In recent years, the development of x-ray free-electron lasers (XFELs) [1] driven by a conventional large-scale radio-frequency accelerator becomes increasingly mature, especially with the great success of several operational facilities around the world [2-5]. Due to the excellent features such as short wavelength, short pulse duration, high brightness, coherence, quasimonochromaticity and continuously tunable frequency, FEL radiation is widely used in the fields of biology, chemistry, materials science and so on [6,7]. With increasing development of the ultrafast sciences, a more compact high-brilliance light source with a reduced cost and size is highly desired, e.g. a tabletop XFEL $[8,9]$. A compact free-electron laser driven by laser plasma accelerator has the potential to achieve such a goal [10-12].

The laser plasma accelerator utilizes a powerful laser to drive plasma gas to produce a few hundred $\mathrm{MeV}$ to a few $\mathrm{GeV}$ energy electron beam with high accelerating gradient of a few hundred GV/m [13-18]. Typically, the laser-driven plasma accelerator (LPA) beam has unique properties that the peak current is up to tens of $\mathrm{kA}$, the pulse duration is on the order of femtosecond, and the normalized transverse emittance is below $1 \mu \mathrm{m}$. With these features, some great efforts have been made by several teams to pave the road to the LPA-driven FEL facilities [19-21]. For instance, the researchers in Shanghai Institute of Optics and Fine Mechanics (SIOM) have successfully developed a cascaded

Published by the American Physical Society under the terms of the Creative Commons Attribution 3.0 License. Further distribution of this work must maintain attribution to the author $(s)$ and the published article's title, journal citation, and DOI.
LPA, obtained quasimonoenergetic electron beams with the energy up to $1.3 \mathrm{GeV}$ [21-23], and been working on FEL realization [24].

The major problems of the LPA beam for FEL radiation are the large initial divergence (up to $1 \mathrm{mrad}$ ) and the relatively large energy spread $(\sim 1 \%)$ which drastically increase the difficulty of transporting the LPA beam from accelerator to radiator and cause FEL radiation gain degradation. In the FEL community, a transverse gradient undulator (TGU) was proposed to minimize the energy spread effect leading to an improvement of FEL gain and radiation power substantially [25]. Consequently, for the realization of LPA-driven FEL based on TGU, it is crucial to design a beam transport system from LPA to TGU.

In this paper, a promising scheme is proposed, including the beam transport line design and the FEL radiation optimization. In Sec. II, FEL generation with a large energy spread beam is discussed, focusing on the TGU FEL. Section III analyzes physics of beam transport from a LPA to a TGU, including chromatic emittance growth, nonlinear dispersion generation, longitudinal bunching lengthening and the corresponding solutions. In Sec. IV, a realistic beam transport line is proposed with a specific example of a $380 \mathrm{MeV}$ LPA beam and the particle tracking is also presented. In Sec. V, FEL simulations based on a TGU are carried out to achieve FEL gain and to demonstrate the feasibility of the beam transport system. Concluding remarks are given in Sec. VI.

\section{FEL GENERATION FOR LARGE ENERGY SPREAD}

As is well known, the LPA beam has a rather large relative energy spread, typically at the several percent 
levels. According to the fundamental FEL resonant condition, a relativistic electron beam propagating through the undulator will emit on-axis radiation with the wavelength of

$$
\lambda_{s}=\frac{\lambda_{u}}{2 \gamma_{0}^{2}}\left(1+\frac{K_{0}^{2}}{2}\right)
$$

where $\gamma_{0}$ is the relativistic electron energy, $\lambda_{u}$ and $K_{0}$ are the undulator period and the dimensionless undulator parameter, respectively. In the high-gain regime, it is convenient to introduce the dimensionless Pierce parameter $\rho$ which is given by [1]

$$
\rho=\frac{1}{\gamma_{0}}\left[\frac{1}{16} \frac{I_{0}}{I_{A}}\left(\frac{K_{0}[J J]}{\sigma_{x} k_{u}}\right)^{2}\right]^{1 / 3},
$$

where $I_{0}$ is the peak current, $I_{A} \approx 17 \mathrm{kA}$ is the Alfvén current, $\sigma_{x}$ is the mean rms transverse beam size in the undulator, $k_{u}=2 \pi / \lambda_{u}$ is the wave vector of the undulator, and the $J J$ field-coupling factor is defined as $[J J]=$ $\left[J_{0}(\xi)-J_{1}(\xi)\right]$ with $\xi=K_{0}^{2} /\left(4+2 K_{0}^{2}\right)$ for a planar undulator. Due to the large energy spread of the beam, it will induce a large spread of the resonant wavelength and degrade the FEL gain. The 1D FEL gain length including the slice energy spread is written as

$$
L_{g} \approx \frac{\lambda_{u}}{4 \pi \sqrt{3} \rho}\left(1+\frac{\sigma_{\delta}^{2}}{\rho^{2}}\right)
$$

with $\sigma_{\delta}$ the FEL slice energy spread, from which we can see that FEL power gain length is severely affected by the energy spread with a quadratic relation, especially $\sigma_{\delta}>\rho$.

As far as we know, there are two methods proposed to increase the FEL performance directing at large energy spread beam, i.e., decompressing the beam [26] and utilizing a TGU as a radiator [25].

The decompression method uses a range of dipoles (chicane) to decompress the electron bunch longitudinally. In this way it reduces the FEL slice energy spread at the cost of sacrificing peak current though. For example, combining chromaticity manipulation to decompression in Ref. [20], some theoretical analysis and simulations have been presented recently.

The TGU method was introduced to reduce the FEL sensitivity to electron energy variations for FEL oscillators in early years [27], and recently was extended to the highgain FEL regime, for instance, enhancing the FEL interaction for LPA-driven FEL [25]. According to the TGU theory, the horizontal dispersion $D$ of electron beam and the transverse gradient $\alpha=d K /\left(d x K_{0}\right)$ of the undulator satisfy the function

$$
D=\frac{2+K_{0}^{2}}{\alpha K_{0}^{2}}
$$

where $K$ is the undulator parameter along the $\mathrm{x}$ axis with $K=K_{0}$ at $x=0$, so that approximately every electron satisfies the resonant condition [Eq. (1)]. An effective energy spread can be introduced to replace the intrinsic large energy spread at the cost of horizontal beam size. For the optimal transverse dispersion factor, it has a minimum gain length approximately as [28]

$$
L_{g, \min }^{T} \approx \frac{4}{3^{3 / 4}} \frac{\lambda_{u}}{4 \pi \sqrt{3} \rho}\left(\frac{\sigma_{\delta}}{\rho}\right)^{1 / 2} .
$$

Hence, with the appropriate parameters the TGU gain length increases as the square root of the intrinsic energy spread, superior to the normal undulator gain length as given in Eq. (3), which also has the same expression as the minimum gain length of the optimal decompression factor [28].

However, the decompression method generates a long bunch with a subsequent energy chirp that will reduce the temporal coherence and increase the FEL bandwidth. On the other hand, the TGU method has a number of potential advantages, including maintaining the ultrashort LPA bunch length and high peak current, providing narrower radiation bandwidth with higher saturation power, stabilization of wavelength fluctuation, and enabling direct or self-seeding. One potential disadvantage of the TGU method is the loss of transverse coherence, yet it can be improved by seeding. Therefore, we consider the TGU method with SASE and direct seeding to design a LPA-driven FEL in this paper.

\section{BEAM OPTICS ANALYSIS OF THE LPA BEAM}

Different from a conventional beam, the large divergence and the large energy spread of the LPA beam would increase the difficulty of beam optics design drastically. We present analysis of these adverse factors and give the corresponding solutions in this section.

\section{A. Emittance growth and nonlinear dispersion effect}

Since the initial beam divergence $\sigma_{x^{\prime}}$ is rather large while the initial beam size $\sigma_{x}$ is rather small, the beam will expand rapidly once it exits plasma into free space. Let us take an example of a typical LPA beam with $\sigma_{x}=1 \mu \mathrm{m}$ and $\sigma_{x^{\prime}}=1 \mathrm{mrad}$, and then a drift distance of $10 \mathrm{~cm}$ would increase the beam size to $100 \mu \mathrm{m}$.

To effectively control the beam expansion and provide beam focusing, high gradient quadrupoles are required to be as close as possible to the LPA exit. For a quadrupole, the linear transport matrix is 


$$
\begin{aligned}
{\left[\begin{array}{c}
x \\
x^{\prime}
\end{array}\right] } & =\left[\begin{array}{cc}
\cos \left(\sqrt{\kappa_{0}} s\right) & \frac{1}{\sqrt{\kappa_{0}}} \sin \left(\sqrt{\kappa_{0}} s\right) \\
-\sqrt{\kappa_{0}} \sin \left(\sqrt{\kappa_{0}} s\right) & \cos \left(\sqrt{\kappa_{0}} s\right)
\end{array}\right]\left[\begin{array}{l}
x_{0} \\
x_{0}^{\prime}
\end{array}\right] \\
& \approx\left[\begin{array}{cr}
1 & 0 \\
-\kappa_{0} s & 1
\end{array}\right]\left[\begin{array}{l}
x_{0} \\
x_{0}^{\prime}
\end{array}\right]
\end{aligned}
$$

with $\kappa_{0}$ and $s$ its focusing strength and focusing length, respectively. With a reference momentum $p_{0}$ we consider an electron with energy deviation $\delta$, thus the quadrupole kick experienced by the electron can be expressed as

$\kappa=\frac{e}{p} \frac{\partial B_{y}}{\partial x}=\frac{e}{p_{0}(1+\delta)} \frac{\partial B_{y}}{\partial x}=\kappa_{0} \frac{1}{1+\delta} \approx \kappa_{0}(1-\delta)$.

This indicates that the electron would receive an additional kick related to its energy deviation from the reference energy. To analyze the effect on beam emittance, one can calculate the following three rms relevant quantities as

$$
\begin{aligned}
\left\langle x^{2}\right\rangle= & \left\langle x_{0}^{2}\right\rangle \\
\left\langle x^{\prime 2}\right\rangle= & \kappa_{0}^{2} s^{2}\left\langle x_{0}^{2}\right\rangle\left\langle 1-2 \delta+\delta^{2}\right\rangle+\left\langle x_{0}^{\prime 2}\right\rangle \\
& -2 \kappa_{0} s\left\langle x_{0} x_{0}^{\prime}\right\rangle\langle 1-\delta\rangle \\
\left\langle x x^{\prime}\right\rangle^{2}= & \left\langle x_{0} x_{0}^{\prime}\right\rangle^{2}+\kappa_{0}^{2} s^{2}\left\langle x_{0}^{2}\right\rangle^{2}\langle 1-\delta\rangle^{2} \\
& -2 \kappa_{0} s\left\langle x_{0} x_{0}^{\prime}\right\rangle\left\langle x_{0}^{2}\right\rangle\langle 1-\delta\rangle .
\end{aligned}
$$

If one only considers the monochromatic beam with any energy deviation $\delta$, the emittance keeps the same value with the initial, i.e. $\varepsilon_{\delta}^{2}=\varepsilon_{0}^{2}$. However, to integrate $\delta$ over the range of the energy spread, the total emittance is given by

$$
\varepsilon^{2}=\varepsilon_{0}^{2}+\sigma_{\delta}^{2} \kappa_{0}^{2} s^{2} \sigma_{x}^{4}
$$

This indicates that the beam suffers an additional emittance growth related to the beam energy spread, beam size and quadrupole strength [29]. To take the above expanded beam with $\sigma_{x}=100 \mu \mathrm{m}$ as an example, a quadruple with $\kappa_{0}=$ $100 \mathrm{~m}^{-2}$ and $s=0.1 \mathrm{~m}$ would contribute a comparable emittance growth as the initial emittance, which would affect the beam transport and FEL radiation.

In addition to the emittance growth, a strong quadrupole also introduces a nonlinear effect on the beam dispersion. Using Eqs. (6) and (7) which are applicable to dispersion evolution, the transport of beam dispersion through a quadrupole can be expressed as

$$
D=D_{0}, \quad D^{\prime}=D_{0}^{\prime}-D_{0} \kappa_{0}(1-\delta)
$$

which suggests the dispersion kick applied to electrons is dependent on electron energy deviation. At the downstream of the quadrupole, the additional kick is also transferred to additional dispersion $\Delta D(\delta)$. In the TGU method, a linear $x-p$ correlation is introduced as $x=D \delta$. Therefore, with $D=D_{0}+\Delta D(\delta)$, a nonlinear term is introduced to the final $x-p$ correlation, which would cause some offmomentum electrons to fall out of resonant and hence degrade the TGU gain.

\section{B. Canceling chromatic emittance and nonlinear dispersion}

With the present chromatic emittance and nonlinear dispersion, it is necessary to include sextupoles to correct these effects. To consider a sextupole attached at the downstream of the quadrupole dispersion included, the kick strength of sextupole is

$$
\Delta \kappa=\frac{1}{(B \rho)_{0}} \frac{\partial B_{y}}{\partial x}=\lambda x=\lambda \delta D
$$

with $\lambda$ the magnetic field strength of the sextupole. Here we assume that $D=D_{0}$ since the dispersion gradient variation is not transferred to a dispersion variation right after the quadrupole. In thin lens approximation, the linear transport matrix is

$$
M_{s}=\left[\begin{array}{cc}
1 & 0 \\
-\lambda \delta D_{0} s & 1
\end{array}\right] \text {. }
$$

Combining it with the quadrupole matrix, we have

$$
M=M_{s} M_{Q}=\left[\begin{array}{cc}
1 & 0 \\
-\kappa_{0} s+\delta s\left(\kappa_{0}-\lambda D_{0}\right) & 1
\end{array}\right] .
$$

It is straightforward to obtain the beam emittance as

$$
\varepsilon^{2}=\varepsilon_{0}^{2}+\sigma_{\delta}^{2} s^{2} \sigma_{x}^{4}\left(\kappa_{0}-\lambda D_{0}\right)^{2} .
$$

It is illustrated that the chromatic emittance can be eliminated by the proper choice of the sextupole strength. Note that since the chromatic emittance exists in both $x$ and $y$ planes, it is necessary to include a set of sextupoles to perform the correction in both planes.

In term of dispersion transport, similar as above, we can obtain

$D=D_{0}, \quad D^{\prime}=D_{0}^{\prime}-D_{0}\left[\kappa_{0} s+\delta s\left(\kappa_{0}-\lambda D_{0}\right)\right]$.

This indicates that with the appropriate parameters the nonlinear $x-p$ correlation can also be canceled along with chromatic emittance.

\section{Bunch lengthening}

To introduce the required dispersion for the TGU radiation and chromatic correction, bending elements should be used. Generally, a dog-leg composed of two dipoles can be adopted, but yet it has large transport elements $R_{52}=L_{D} \theta$ and $R_{56}=L_{D} \theta^{2}$ with $L_{D}$ the 
separation distance of dipoles and $\theta$ the deflection angle. The electron longitudinal position transports as

$$
z=R_{51} x_{0}+R_{52} x_{0}^{\prime}+z_{0}+R_{56} \delta,
$$

where $z_{0}$ is the particle longitudinal position, $x_{0}$ and $x_{0}^{\prime}$ are the transverse position and its slope before the bending magnets, respectively. For the LPA beam with a large energy spread and a short bunch length, the bunch length can be increased significantly with such a transport system. As a result, the peak current will be reduced leading to a degradation of FEL gain. References [18,30,31] adopt a dog-leg scheme with quadrupoles inserted. Such a scheme reduces the efficiency of the TGU and enhances the difficulty of beam optics.

We proposed a single dipole scheme to provide the necessary dispersion. Taking into account the combination of one dipole and several quadrupoles, the electron longitudinal position evolution can be calculated as

$$
z=z_{0}-\theta x_{0}-\frac{L_{B} \theta}{2} x_{0}^{\prime}-\frac{L_{B} \theta^{2}}{6} \delta
$$

with $L_{B}$ the deflection length of the dipole. Note that all the terms only depend on the single dipole. By comparison, $R_{52}$ and $R_{56}$ are much smaller than that of a dog-leg. Although $R_{51}$ is larger than a dog-leg, this term can be minimized by placing the dipole at a beam waist position. Therefore, the LPA beam lengthening can be minimized and the high peak current can be reserved as much as possible.

\section{BEAM TRANSPORT SYSTEM}

According to the above analysis, we present our scheme of the beam transport system by employing the attainable LPA beam parameters from SIOM [23] listed in Table I.

The beam transport system is shown in Fig. 1, which includes an initial strong focusing stage, a subsequent optics matching stage, and an associated chromatic correction stage. Similar to other schemes [20,32], the strong focusing stage is composed of three compact and high gradient quadrupoles, placing close to the plasma jet position of the LPA after a drift distance of $10 \mathrm{~cm}$. Considering the SIOM LPA facility limited by the vacuum chamber structure, the former two quadrupoles are put inside the chamber with a gradient up to $150 \mathrm{~T} / \mathrm{m}$ and the third one is outside with gradient of $80 \mathrm{~T} / \mathrm{m}$. The employment of electromagnet quadrupoles other than a permanent magnet enables adjustable matching into the subsequent stage for different central energy. The optics matching stage includes one dipole with a deflection angle of $\theta=0.1 \mathrm{rad}$ to introduce dispersion and three quadrupoles with a gradient of $\sim 80 \mathrm{~T} / \mathrm{m}$ to match the Twiss parameters and dispersion required by the TGU FEL. The correction stage
TABLE I. The parameters of the LPA beam for SIOM facility studies.

\begin{tabular}{lccc}
\hline \hline Parameter & Symbol & Value & Unit \\
\hline Beam Energy & $\gamma_{0} m c^{2}$ & 380 & $\mathrm{MeV}$ \\
Rms Energy Spread & $\sigma_{\delta}$ & $1 \%$ & $/$ \\
Normalized rms Emittance & $\gamma_{0} \varepsilon_{x, y}$ & $0.1-1$ & $\mu \mathrm{m}$ \\
Rms Beam Size & $\sigma_{x, y}$ & $1-2$ & $\mu \mathrm{m}$ \\
Rms Beam Divergence & $\sigma_{x, y}^{\prime}$ & $0.1-1$ & $\mathrm{mrad}$ \\
Charge & $Q$ & 80 & $\mathrm{pC}$ \\
FWHM Bunch length & $L_{s}$ & 2.4 & $\mu \mathrm{m}$ \\
Peak Current & $I_{0}$ & 10 & $\mathrm{kA}$ \\
\hline \hline
\end{tabular}

composed of three sextupoles is inserted into the matching stage with the strength of several thousand $\mathrm{T} / \mathrm{m}^{2}$.

For the beam matching, we used MAD [33] to simulate the beam optics setting initial Twiss parameters as $\beta_{0}=0.001 \mathrm{~m}$ and $\alpha_{0}=0$ (beam waist). The final matching conditions during the undulator are Twiss $\bar{\beta}_{x, y} \approx 3$, dispersion $D_{x}=-21 \mathrm{~mm}$ and its slope $D_{x}^{\prime}=0$, as shown in Fig. 2. Note that $\beta_{x}, \beta_{y}$ and $D_{x}$ are shown for only the central energy particles. In terms of the off-momentum particles, there are some small deviations due to the residual chromatic emittances and nonlinear dispersion despite the sextupole correction. Meanwhile, we also matched a beam waist in the $x$ direction at the position of the single dipole to restrain bunch lengthening effectively.

To verify the above theoretical analysis and further investigate the beam performance with the designed scheme, we conducted particle tracking using Elegant [34] with a beam of $380 \mathrm{MeV}$ energy, $0.45 \mu \mathrm{m}$ normalized emittance and $0.3 \mathrm{mrad}$ divergence. It is noted that we assume a Gaussian beam without any initial energy correlation since the bunch length is comparable to the FEL cooperation length. In Fig. 3, the evolutions of emittances and beam sizes with and without sextupole correction are presented. With the sextupoles, the transverse emittance growth is significantly reduced and the transverse beam size is well controlled after the beam transport system of less than 3 meters. The sextupoles suppress the emittance growth to a large degree but not completely, because of the overlarge chromaticity and the high order effects of sextupoles. Figure 4 shows the $x-\gamma$ phase space at the entrance of the undulator without [4(a)] and with [4(b)] sextupole correction, at the position of $z=2.7 \mathrm{~m}$ in Fig. 2(c). It can be observed that a nonlinear curvature of $x-\gamma$ correlation is effectively eliminated with only the required linear component left when these sextupoles are employed. In terms of the longitudinal phase space, the FWHM bunch length is increased from 2.4 to $4 \mu \mathrm{m}$ with the peak current dropping from 10 to $\sim 5 \mathrm{kA}$. The bunch length is still comparable to the cooperation length.

To understand the beam evolution along the undulator section, the transverse phase spaces and the $x-\gamma$ 


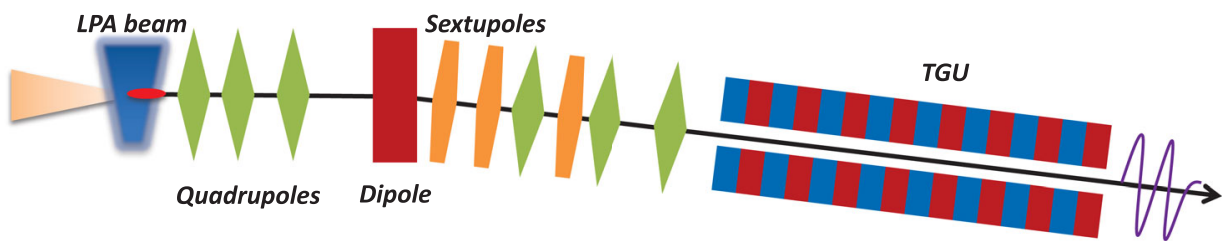

FIG. 1. Scheme of the proposed LPA beam transport system combining to TGU.

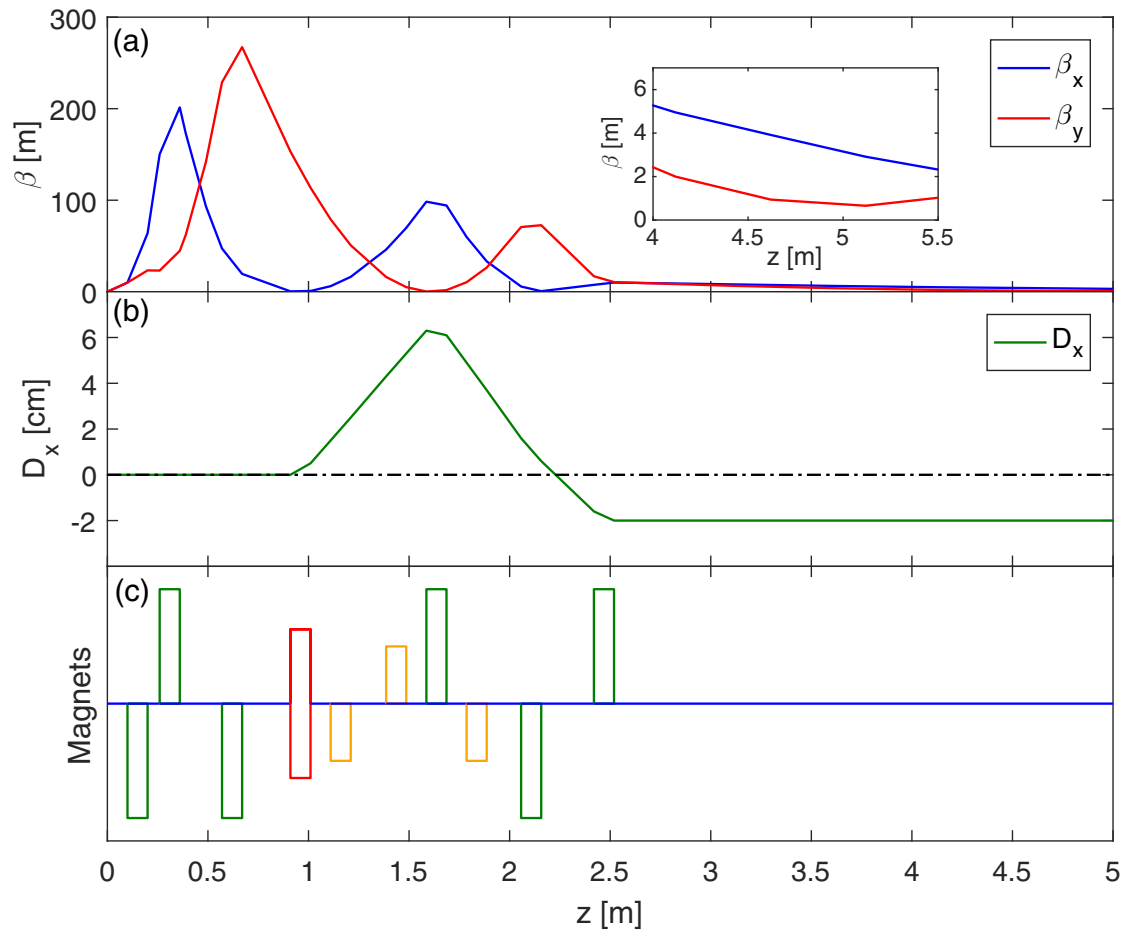

FIG. 2. Beta functions (a) and dispersion function (b) of the reference energy along the beam line. (c) Layout of the beam transport system including one dipole (red), six quadrupoles (green) and three sextupoles (orange). After the position of $\mathrm{z}=2.7 \mathrm{~m}$, the drift space is used for the TGU radiation.
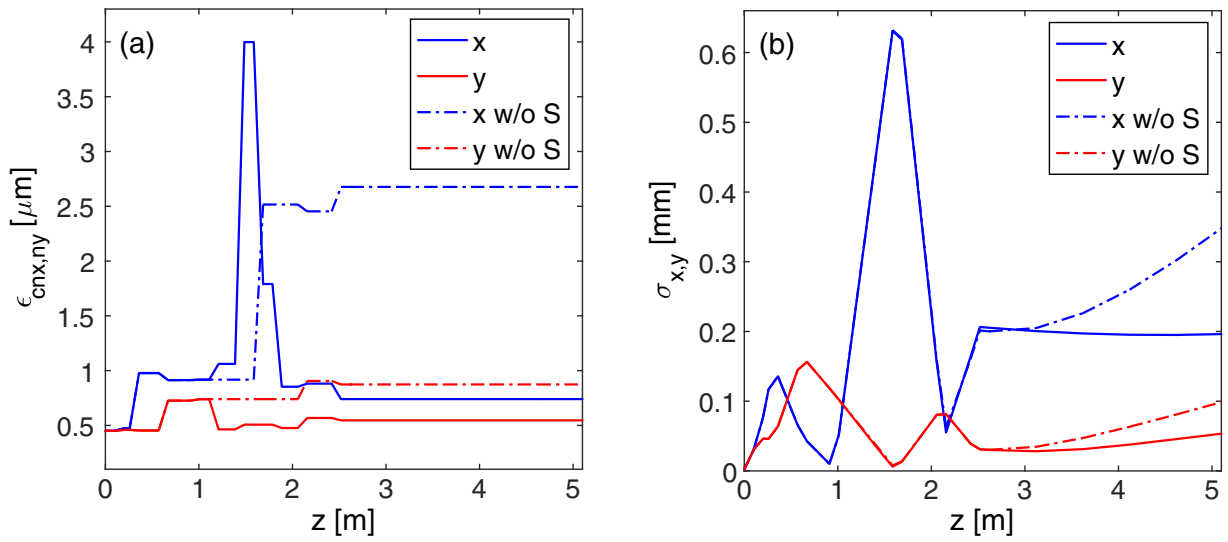

FIG. 3. Evolutions of the LPA beam properties with (solid) and without (dash-dotted) chromatic correction, respectively. (a) Evolutions of the normalized projected rms transverse emittances. (b) Evolutions of the rms beam sizes along the beam line. Note: the emittances of $x$ are without dispersion contribution. 

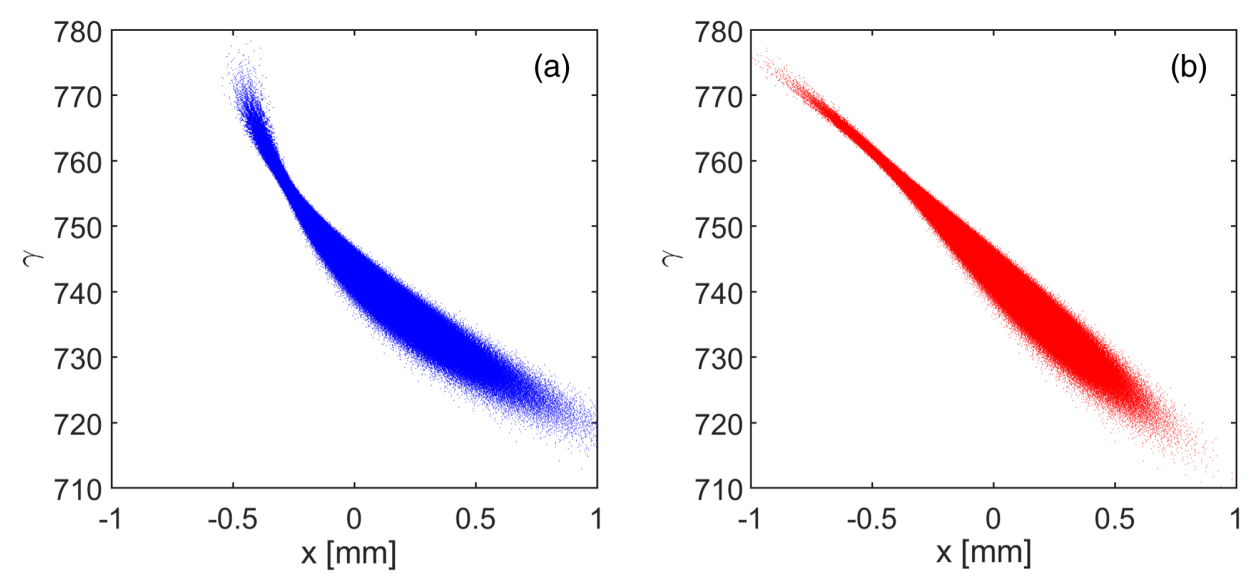

FIG. 4. Dispersion distributions at the entrance of the TGU undulator. (a) Nonlinear distribution of $x-\gamma$ phase space through the focusing system including dipole and quadrupoles. (b) Quasilinear distribution of $x-\gamma$ phase space with additional sextupoles.

correlation of the beam at the entrance of the undulator and after a three-meter drift are shown in Fig. 5. Both of the transverse phase space plots show the beam sizes change slowly (a dispersion component along the $x$ axis is included). From the $x-\gamma$ correlation, we note that the dispersion is maintained in the three-meter drift. The increase in the uncorrelated part in the $x-\gamma$ distribution
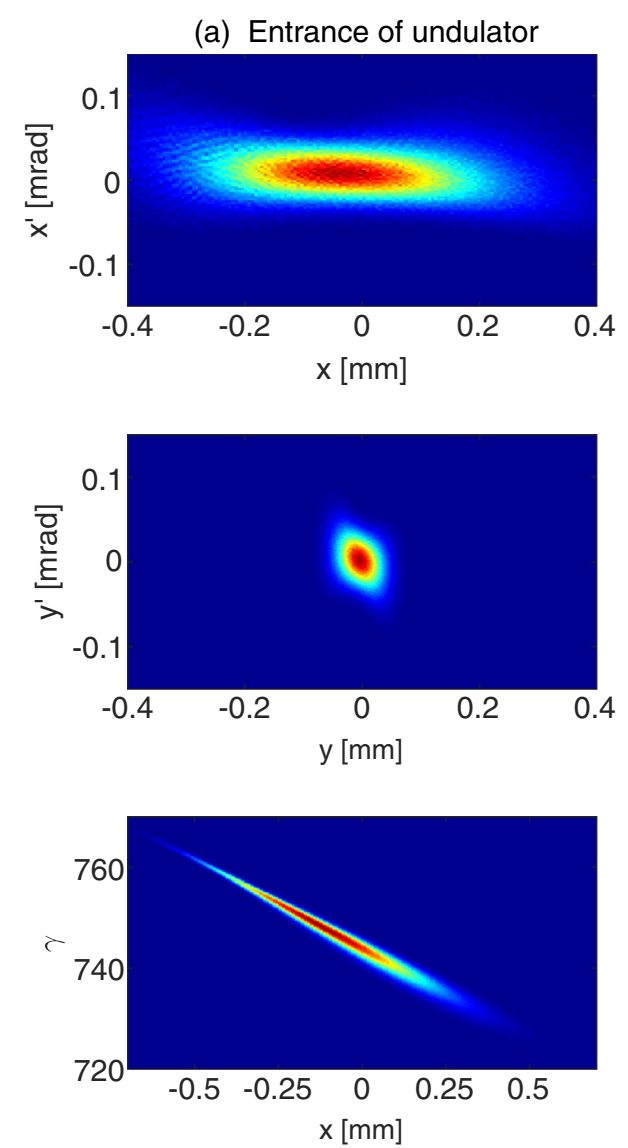

is due to the horizontal betatron beam size increase in this drift. Therefore, the compact optics provides the necessary beam properties for the TGU FEL. Finally, the results of particle tracking at the entrance of the TGU undulator are summarized in Table II.

We further conducted simulations in order to take into account collective effects such as space charge and
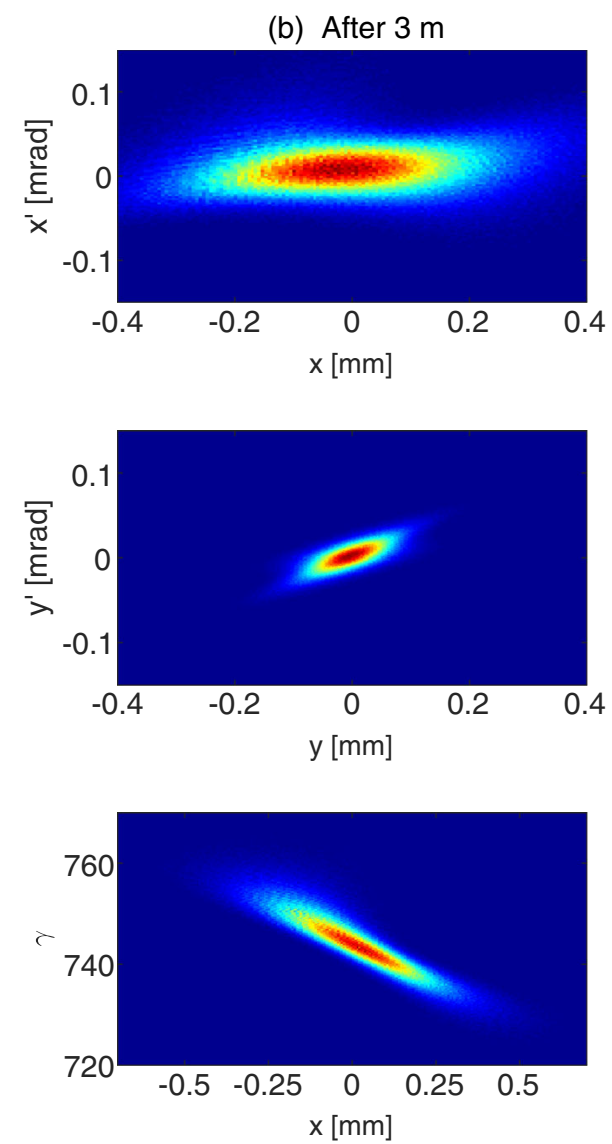

FIG. 5. Transverse phase space distributions and dispersion distribution at the entrance of the TGU section (left) and after a following $3 \mathrm{~m}$ drift distance (right). 
TABLE II. The results of the LPA beam tracking.

\begin{tabular}{lccc}
\hline \hline Parameter & Symbol & Value & Unit \\
\hline Beam Energy & $\gamma_{0} m c^{2}$ & 380 & $\mathrm{MeV}$ \\
Normalized rms Emittance & $\gamma_{0} \varepsilon_{x} / \gamma_{0} \varepsilon_{y}$ & $0.72 / 0.53$ & $\mu \mathrm{m}$ \\
Rms Beam Size & $\sigma_{x} / \sigma_{y}$ & $200 / 40$ & $\mu \mathrm{m}$ \\
Dispersion & $D$ & -21 & $\mathrm{~mm}$ \\
FWHM Bunch length & $L_{s}$ & 4 & $\mu \mathrm{m}$ \\
Peak Current & $I_{0}$ & 5.4 & $\mathrm{kA}$ \\
\hline \hline
\end{tabular}

coherent synchrotron radiation (CSR). For the space charge effects, we tracked the initial LPA beam through a segment of the beam line using Astra [35]. The result is consistent with Elegant simulations without space charge. This indicates that the space charge effect is not significant despite the micron level beam size and the kA level beam current, since the beam has a rather high energy and a large divergence. For the CSR effects, we used Elegant with a 1D CSR model $[36,37]$ to track the beam through the beam transport system. As shown in Fig. 6, we presented the
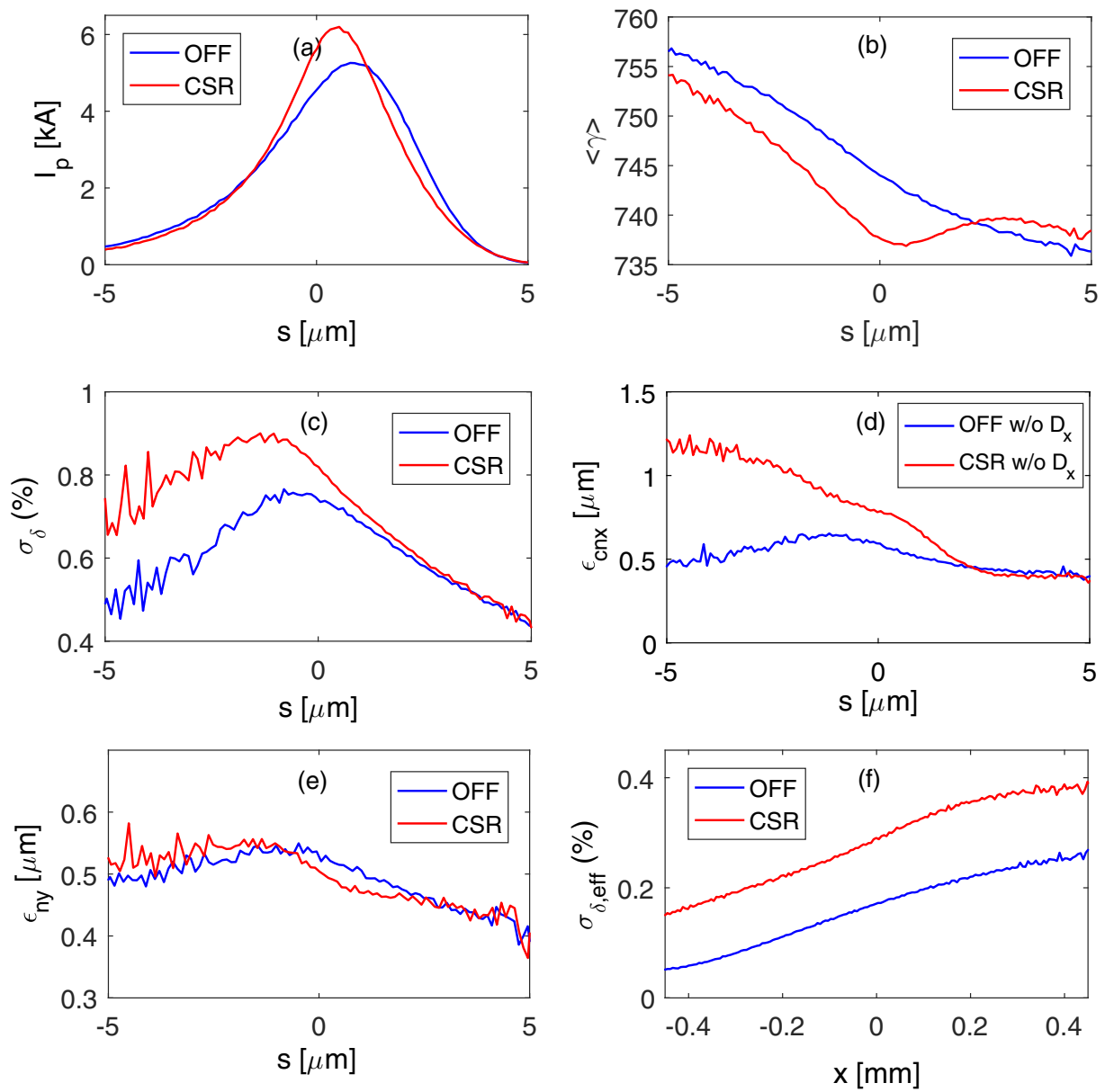

FIG. 6. LPA beam properties at the entrance of the TGU with (red) and without (blue) CSR effects, including the current distribution (a), the slice average energy (b), the slice energy spread (c), the slice transverse emittances (d) and (e), and the x-slice effective energy spread (f). Part (d) excludes dispersion contribution. 
TABLE III. The parameters of TGU and seed laser for FEL simulation.

\begin{tabular}{lccc}
\hline \hline Parameter & Symbol & Value & Unit \\
\hline Undulator period & $\lambda_{u}$ & 2 & $\mathrm{~cm}$ \\
Undulator parameter & $K_{0}$ & 1.15 & $/$ \\
Undulator transverse gradient & $\alpha$ & -120 & $\mathrm{~m}^{-1}$ \\
Seed laser power & $P_{0}$ & 1 & $\mathrm{MW}$ \\
Rayleigh length & $L_{R}$ & 2 & $\mathrm{~m}$ \\
Resonant wavelength & $\lambda_{s}$ & 30 & $\mathrm{~nm}$ \\
Pulse duration (FWHM) & $\tau_{s}$ & $\sim 8$ & $\mathrm{fs}$ \\
\hline \hline
\end{tabular}

is expected to be obtained from a $30 \mathrm{~nm}$ high-harmonic generation source with $1 \mathrm{MW}$ power [39]. The FWHM pulse duration is $\sim 8 \mathrm{fs}$ and the rms spot size is $100 \mu \mathrm{m}$ at the entrance of the undulator. As a comparison, we simulated SASE and seeded configurations with a normal planar undulator using an optimized beam with the same parameters (Twiss parameters, emittances, current, etc.) as the tracked beam without the horizontal dispersion.

Other than the normal undulator, the TGU undulator would induce an additional dispersion variation and natural focusing in the $x$ direction. For TGU magnets, a corrector magnetic field is attached to counteract the horizontal trajectory deviation, which can be simplified to the first order of $x$ dependence and expressed as [40]

$$
B_{e}=\frac{m c}{e} \frac{\alpha K_{0}^{2}}{2 \gamma_{0}}\left(1-\frac{x}{D_{0}}\right)
$$

Taking into account a constant static magnetic field, and neglecting the second term of this equation, the horizontal deviation will be related to energy and induce dispersion variation. According to the parameters with $D_{0}=-21 \mathrm{~mm}$ and $D_{0}^{\prime}=0$, through a $5 \mathrm{~m}$ distance, TGUs induce a dispersion variation about $1.8 \mathrm{~mm}$ which is 1 order of magnitude smaller than the value $D_{0}$. When the second term is included in the corrector field, dispersion variation is canceled and yet it induces the betatron motion and a weak horizontal focusing. For simplicity, we ignored these effects approximately in the FEL simulation.

The evolutions of the FEL peak power along the undulator are illustrated in Fig. 7. For the seeded FEL, the TGU case excluding CSR (solid blue) has a saturation power up to $4 \mathrm{GW}$ and the case with CSR (solid red) is also up to $\sim 2 \mathrm{GW}$, while the power of the planar undulator case (solid green) is below $100 \mathrm{MW}$. Thanks to the compensation of energy spread by the TGU, the TGU cases can generate stronger bunching before the radiation slips out of the electron beam. The CSR effects do not reduce the saturation power drastically but increase the saturation length. It additionally leads to the redshift of the radiation wavelength due to the energy loss, as shown in Fig. 8. For the SASE FEL, the final power is relatively low due to the large slippage effect. Nevertheless, the exponential gain can

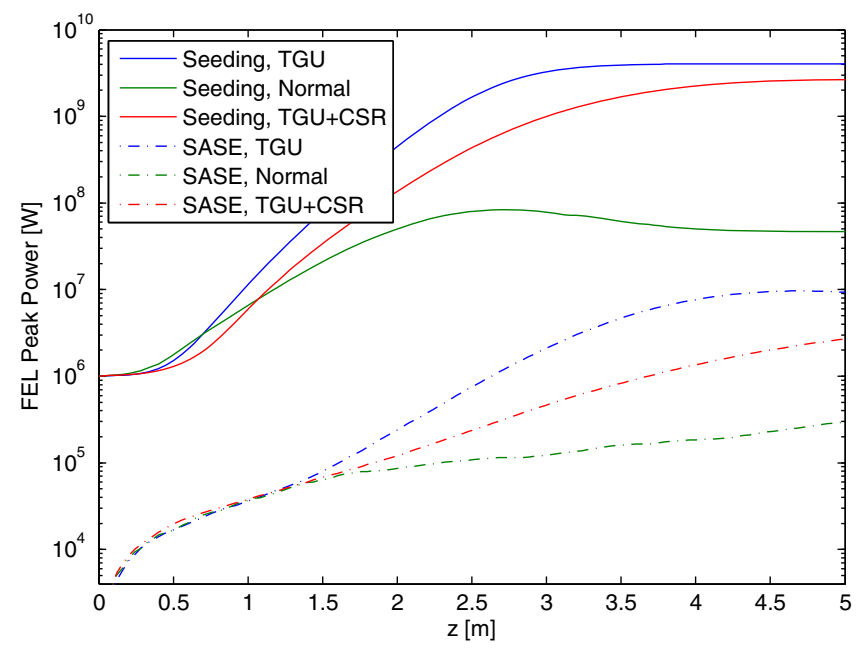

FIG. 7. SASE (dash dotted) and seeded (solid) FEL peak power growth based on TGU cases without CSR (blue), with CSR (red) and on normal undulator case (green) along the undulator.

still be achieved in the TGU cases. In the frequency domain, the bandwidths in the TGU cases are much smaller than the normal undulator one owing to the energy spread compensation. Furthermore, since the bunch length is comparable to the coherence length, the spectra in the TGU cases show single coherent spikes.

In general, for an ideal LPA beam with the emittances of $0.45 \mu \mathrm{m}$ and the peak current of $10 \mathrm{kA}$, the peak power in the SASE configuration within a 5-meter TGU can reach about $100 \mathrm{MW}$ to $1 \mathrm{GW}$. However, from the above start-toend (S2E) simulation, we see that the LPA beam quality will be deteriorated, and the FEL power is about 1 to 2 orders of magnitude lower than the ideal case. This clearly illustrates the importance of including the beam dynamics effects in transporting the LPA beam to the TGU.

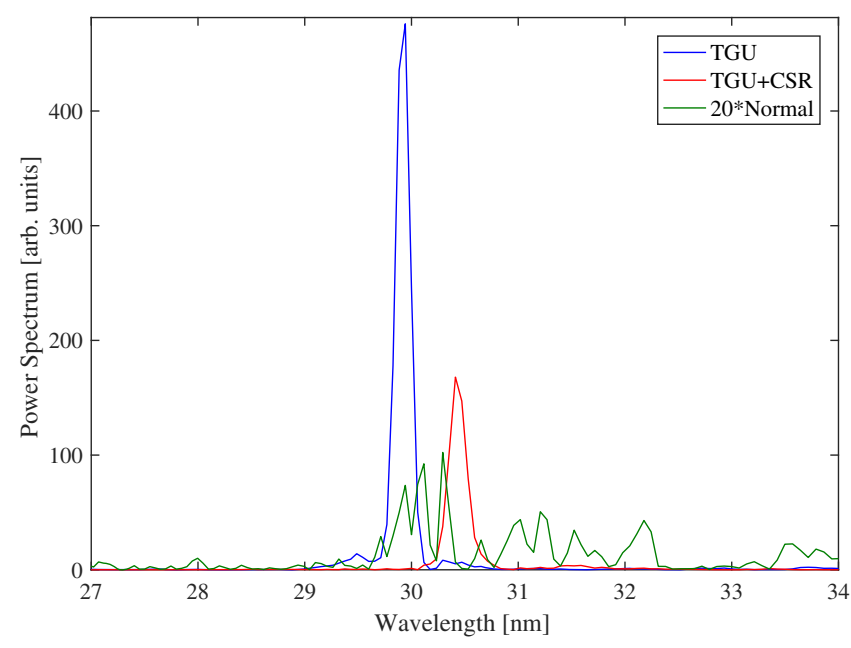

FIG. 8. Typical single-shot radiation spectrum of SASE FEL for the TGU cases and the normal undulator case through a $5 \mathrm{~m}$ distance. 

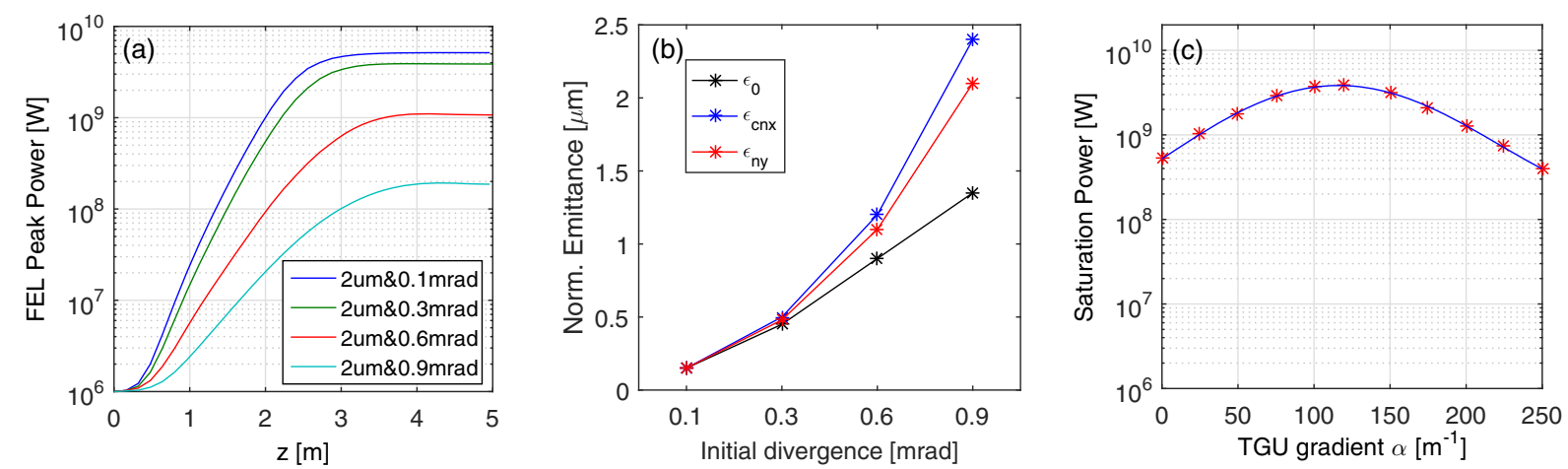

FIG. 9. (a) Seeded FEL power growth for the LPA beam with different initial divergence (emittance). (b) Normalized sliced emittance growth versus initial divergence. The slice is at the longitudinal position of the current peak. (c) Saturation power of seeded FEL as a function of TGU gradient for the tracked beam with a fixed dispersion of $21 \mathrm{~mm}$.

There are several practical issues to be considered here. One is the variation of the initial beam divergence at the scale of $0.1-1 \mathrm{mrad}$, as illustrated in Table I. Figure 9(a) shows the radiation power of the seeded FEL along a 5-m TGU undulator with a fixed rms beam size of $2 \mu \mathrm{m}$ and variable rms divergence (i.e. different emittance) using the $\mathrm{S} 2 \mathrm{E}$ simulations. The corresponding normalized sliced emittance growth at the position of peak current are also presented in Fig. 9(b). The seeded FEL power drops 1 to 2 orders when the beam divergence is increased from 0.1 to $0.9 \mathrm{mrad}$, but still maintains considerable gain. In addition, it is worth noting that the resonance relation of Eq. (4) is not always satisfied experimentally between beam dispersion and TGU gradient, which leads a degradation of the FEL gain [41]. Hence, we show in Fig. 9(c) the simulated saturation power with a different TGU gradient at a fixed dispersion. The saturation power variation is within 1 order of magnitude over a wide range of the TGU gradient.

\section{CONCLUSION}

In this paper, we have proposed a compact scheme of the LPA beam transport system combining with the TGU radiator to achieve significant FEL gain, in which both theoretical analysis and numerical simulations were performed to demonstrate its validity. The theoretical analysis shows that the strong focusing quadrupoles and the chromatic correction sextupoles are the critical components for the beam transport system. Both the increase of the chromatic emittance and the nonlinearity of the dispersion induced by the quadrupoles can be compensated by these sextupoles. We have also demonstrated that the layout of the single dipole case not only simplifies the design of the beam line and facilitates the beam adjustment, but also avoids the bunch lengthening to maintain high peak current and ultrashort radiation pulse. Simulation shows that a significant FEL gain within a short TGU undulator is achievable at a wavelength of $30 \mathrm{~nm}$. Such a beam transport combining with the TGU undulator will be tested at the LPA-FEL facility in SIOM experimentally [24].

\section{ACKNOWLEDGMENTS}

We would like to thank Z. Wang, Y. Ding, W. Qin, C. Feng, H. Deng, B. Liu, G. Wang, and J. Liu for helpful discussions and useful comments. This work is partially supported by the Ministry of Science and Technology of China (Grant No. 2016YFA0401900), the National Natural Science Foundation of China (Grant No. 11127901) and the U.S. National Science Foundation (Grant No. PHY1535215).

[1] Z. Huang and K.-J. Kim, Review of x-ray free-electron laser theory, Phys. Rev. ST Accel. Beams 10, 034801 (2007).

[2] W. Ackermann et al., Operation of a free-electron laser from the extreme ultraviolet to the water window, Nat. Photonics 1, 336 (2007).

[3] P. Emma et al., First Lasing and operation of an ångströmwavelength free-electron laser, Nat. Photonics 4, 641 (2010).

[4] T. Ishikawa et al., A compact X-ray free-electron laser emitting in the sub-ångström region, Nat. Photonics 6, 540 (2012).

[5] E. Allaria et al., Highly coherent and stable pulses from the FERMI seeded free-electron laser in the extreme ultraviolet, Nat. Photonics 6, 699 (2012).

[6] L. Young et al., Femtosecond electronic response of atoms to ultra-intense X-rays, Nature (London) 466, 56 (2010).

[7] H. N. Chapman et al., Femtosecond X-ray protein nanocrystallography, Nature (London) 470, 73 (2011).

[8] C. B. Schroeder, W. M. Fawley, E. Esarey, and W. P. Leemans, Design of an XUV FEL driven by the laserplasma accelerator at the LBNL LOASIS facility, in Proceedings of the 28th International Free Electron Laser Conferences, FEL2006, Berlin, Germany, 2006 (JACoW, Geneva, 2006). p. 455.

[9] F. Gruner et al., Design considerations for table-top, laserbased VUV and X-ray free electron lasers, Appl. Phys. B 86, 431 (2007). 
[10] K. Nakajima, Compact X-ray sources: Towards a table-top free-electron laser, Nat. Phys. 4, 92 (2008).

[11] M. Fuchs et al., Laser-driven soft-X-ray undulator source, Nat. Phys. 5, 826 (2009).

[12] M. E. Couprie, A. Loulergue, M. Labat, R. Lehe, and V. Malka, Towards a free electron laser based on laser plasma accelerators, J. Phys. B 47, 234001 (2014).

[13] T. Tajima and J. M. Dawson, Laser Electron Accelerator, Phys. Rev. Lett. 43, 267 (1979).

[14] E. Esarey, C. B. Schroeder, and W. P. Leemans, Physics of laser-driven plasma-based electron accelerators, Rev. Mod. Phys. 81, 1229 (2009).

[15] V. Malka, Laser plasma accelerators, Phys. Plasmas 19, 055501 (2012).

[16] X. Wang et al., Quasi-monoenergetic laser-plasma acceleration of electrons to $2 \mathrm{GeV}$, Nat. Commun. 4, 1988 (2013).

[17] H. T. Kim, K. H. Pae, H. J. Cha, I. J. Kim, T. J. Yu, J. H. Sung, S. K. Lee, T. M. Jeong, and J. Lee, Enhancement of Electron Energy to the Multi-GeV Regime by a Dual-Stage Laser-Wakefield Accelerator Pumped by Petawatt Laser Pulses, Phys. Rev. Lett. 111, 165002 (2013).

[18] W. P. Leemans et al., Multi-GeV Electron Beam from Capillary-Discharge-Guided Subpetawatt Laser Pulses in the Self-Tripping Regime, Phys. Rev. Lett. 113, 245002 (2014).

[19] C. Widmann et al., First tests of a beam transport system from a laser wakefield accelerator to a transverse gradient undulator, in Proceedings of the 6th International Particle Accelerator Conference, IPAC2015, Richmond, 2015 (JACoW, Geneva, 2015), MOPWA045.

[20] A. Loulergue, M. Labat, C. Evain, C. Benabderrahmane, V. Malka, and M. E. Couprie, Beam manipulation for compact laser wakefield accelerator based free-electron lasers, New J. Phys. 17, 023028 (2015).

[21] J.S. Liu et al., All-Optical Cascaded Laser Wakefield Accelerator using Lionization-Induced Injection, Phys. Rev. Lett. 107, 035001 (2011).

[22] W.-T. Li, W.-T. Wang, J.-S. Liu, C. Wang, Z.-J. Zhang, R. Qi, C.-H. Yu, R.-X. Li, and Z.-Z. Xu, Developments in laser wakefield accelerators: From single-stage to twostage, Chin. Phys. B 24, 015205 (2015).

[23] W. T. Wang et al., High-Brightness High-Energy Electron Beams from a Laser Wakefiled Accelerator via Energy Chirp Control, Phys. Rev. Lett. 117, 124801 (2016).

[24] T. Liu, B. Liu, D. Wang, T. Zhang, Z. Huang, and J. S. Liu, Beam transport line of the LPA-FEL facility based on transverse gradient undulator, in Proceedings of the 7th International Particle Accelerator Conference, IPAC2016, Busan, Korea, 2016 (JACoW, Geneva, 2016), THPMB027.

[25] Z. Huang, Y. Ding, and C. B. Schroeder, Compact X-ray Free-Electron Laser from a Laser-Plasma Accelerator Using a Transverse-Gradient Undulator, Phys. Rev. Lett. 109, 204801 (2012).
[26] A. R. Maier, A. Meseck, S. Reiche, C. B. Schroeder, T. Seggebrock, and F. Grüner, Demonstration Scheme for a Laser-Plasma-Driven Free-Electron Laser, Phys. Rev. X 2 , 031019 (2012).

[27] T. Smith, J. M. J. Madey, L. R. Elias, and D. A. G. Deacon, Redusing the sensitivity of a free-electron laser to electron energy, J. Appl. Phys. 50, 4580 (1979).

[28] C. B. Schroeder, E. Esarey, W. P. Leemans, J. van Tilborg, F. F. Grüner, Y. Ding, and Z. Huang, in Proceedings of the 35th International Free-Electron Laser Conference, FEL2013, New York, 2013 (JACoW, Geneva, 2013), MOPSO69.

[29] J. Buon, Beam phase space and emittance, in CERN Accelerator School: 5th General Accelerator Physics Couse, Jyväskylä, 1992, edited by S. Turner (Geneva, 1994), Vol. I, p. 89 (CERN Accelerator School Report No. CERN 94-01, 1994).

[30] C. Widmann et al., Non-linear beam transport optics for a laser wakefield accelerator, in Proceedings of the 4th International Particle Accelerator Conference, IPAC2013, Shanghai, China, 2013 (JACoW, Geneva, 2013), TUPWO013.

[31] C. Widmann et al., Beam transport system from a laser wakefield accelerator to a transverse gradient undulator, in Proceedings of the 5th International Particle Accelerator Conference, IPAC2014, Dresden, Germany, 2014 (JACoW, Geneva, 2014), THOBA03.

[32] M. P. Anania et al., The ALPHA-X beam line: Toward a compact FEL, in Proceedings of the 31st International Free Electron Laser Conference, FEL2009, Liverpool, 2009 (JACoW, Geneva, 2009), MOPC71.

[33] H. Grote and F. C. Iselin, Report No. CERN/SL/90-13(AP) (Rev. 4), 1995, http://mad.web.cern.ch/mad/mad8/mad8.html.

[34] M. Borland, Argonne National Laboratory Advanced Photon Source Report No. LS-287, 2000.

[35] K. Floettmann, ASTRA, http://www.desy.de/ mpyflo (1999).

[36] M. Borland, Simple method for particle tracking with coherent synchrotron, Phys. Rev. ST Accel. Beams 4, 070701 (2001).

[37] G. Stupakov and P. Emma, SLAC Technical Report No. LCLS TN-01-12, 2001.

[38] S. Reiche, GENESIS 1.3: A fully 3D time-dependent FEL simulation code, Nucl. Instrum. Methods Phys. Res., Sect. A 429, 243 (1999).

[39] J. L. Krause, K. J. Schafer, and K. C. Kulander, High-Order Harmonic Generation from Atoms and Ions in the High Intensity Regime, Phys. Rev. Lett. 68, 3535 (1992).

[40] N. M. Kroll, P. L. Morton, M. N. Rosenbluth, J. N. Eckstein, and J. M. J. Madey, Theory of the transverse gradient wiggler, IEEE J. Quantum Electron. 17, 1496 (1981).

[41] P. Baxevanis, Z. Huang, R. Ruth, and C. B. Schroeder, Eigenmode analysis of a high-gain free-electron laser based on a transverse gradient undulator, Phys. Rev. ST Accel. Beams 18, 010701 (2015). 